noch an, daß bei einem dunkeln Macispulver von sebr hohem Fettgehalt, aber von sonst normaler Beschaffenheit auf das Vorhandensein von Papuamacis zu schließen sei. Nach den von Busse ausgeführten Untersuchungen enthieli die Papuamacis 53,4 bis 55,5\% ätherisches Extrakt (Petrolätherextrakt), wovon $52-54 \%$ reines Fett darstellten. In der Bombaymacis fand Bus se $61-67 \%$ ätherisches Extrakt aber nur $29,6-34,2 \%$ reines Fett; diese Differenz wird durch den hohen Harzgehal hervorgerufen.

\title{
Die Bestimmung des Fettes in Kakao.
}

\author{
Von
}

\section{Aage Kirschner.}

Die Fettbestimmung im Kakao geschieht hauptsächlich durch Extraktion mit Äther und dieses Verfahren liefert sehr genau übereinstimmende Ergebnisse. Weil diese Methode aber eine ziemlich lange Zeit in Anspruch nimmt, habe ich auf Veranlassung von Detlefsen und Meyer's Laboratorium in Kopenhagen versucht, das Gottlieb'sche Verfahren, welches, wie bekannt, für die Fettbestimmung in Milch so vorzügliche Dienste leistet, auch bei Kakaountersuchungen zu verwenden und habe ich auch hier sehr zufriedenstellende Ergebnisse erhalten.

Die Bestimmung wird wie folgt ausgeführt:

Man verwendet einen in halbe Kubikzentimeter genau eingeteilten Meßcylinder von 75 oder $100 \mathrm{ccm}$, welcher von 0 bis 75 nicht kürzer als etwa $30 \mathrm{ccm}$ sein darf, weil sonst die Ablesungen nicht genau genug werden. In den Cylinder werden $20 \mathrm{cem}$ Alkohol von $50 \mathrm{Vol} . \%$ und etwa $1,5 \mathrm{~g}$ Kakao eingetragen.

Der Kakao wird durch Schütteln mit dem Alkohol gut durchfeuchtet, und es werden jetzt genau $25 \mathrm{ccm}$ Äther zugegeben; während 15 Minuten wird ab und zu gut geschüttelt. Darauf werden genau $25 \mathrm{ccm}$ Petroläther (Siedepunkt unter $80^{\circ} \mathrm{C}$ ) zugesetzt und nun wird durch vorsichtige Bewegung des Cylinders alles gemischt. Durch zu starkes Schütteln bildet sich leicht eine sehr schwer trennbare Emulsion. Der Cylinder wird eine Stunde beiseite gestellt; alsdann zieht man mit dem Heber (wie bei den Milchuntersuchungen) genau $45 \mathrm{ccm}$ der ätherischen Lösung ab, verdunstet das Lösungsmittel auf dem Wasserbade, trocknet und wägt das zurückbleibende Fett.

Da das Ablesen der Alkoholoberfläche durch die Gegenwart des Kakao erschwert wird, und weil die gewöhnlichen Mefcylinder nicht ganz genau eingeteilt sind, empfiehlt es sich, ein für allemal das gesamte Volumen der Äthermischung ohne Kakao für jeden Cylinder zu bestimmen. Dies geschieht, wie oben angegeben, nur ohne Zusatz von Kakao. Zu dem so gefundenen Volumen wird das gefundene Fettgewicht, in ccm ausgedrückt, hinzuaddiert und die weitere Rechnung wie gewöhnlich ausgeführt.

Beispiel; In einem Cylinder beträgt das Äther-Petroläther-Volumen 52,4 ccm. Es sind $1,5167 \mathrm{~g}$ Kakao abgewogen, $45 \mathrm{ccm}$ ätherische Lösung abgezogen und $0,2947 \mathrm{~g}$ Fett gefunden. Das gesamte Volumen der ätherischen Fettlösung ist hiernach $52,7 \mathrm{ccm}$ und der Kakao enthält also:

$$
\frac{0,2947 \times 52,7 \times 100}{45 \times 1,5167}=22,67 \% \text { Fett. }
$$


Bei der Untersuchung von fünf verschiedenen Kakaopulvern wurden die folgenden procentualen Fettgehalte gefunden:

\begin{tabular}{c|c|c|c|c|c|c}
\hline Kakaopulver & $\begin{array}{c}\text { Nach dem Extraktions- } \\
\text { Verfahren }\end{array}$ & \multicolumn{3}{|c|}{ Nach dem Gottli e b schen Verfahren } \\
\hline I & 22,61 & 22,68 & 22,56 & 22,60 & 22,64 & 22,70 \\
II & 33,02 & 32,99 & 32,80 & 32,80 & 32,98 & - \\
III & 20,50 & - & 20,47 & 20,45 & 20,44 & 20,48 \\
IV & 19,21 & - & 19,23 & 19,11 & - & - \\
V & 20,31 & 20,28 & 20,27 & 20,33 & 20,29 & -
\end{tabular}

Kopenhagen, 19. März 1906.

\section{Referate.}

\section{Milch und Käse.}

Th. Pfeiffer, A. Einecke und W. Schneider: Über den Einfluß des Asparagins auf die Erzeugung der Milch und ihrer Bestandteile. (Mittlg. der landw. Institute der Kgl. Univ. Breslau, 1905, 3, 179-225.) - Während die Frage nach dem Einfluß des Asparagins als Repräsentanten der Amidverbindungen auf den Eiweißumsatz einigermaßen dahingehend geklärt ist, daß die eiweißsparende Wirkung des Asparagins nur in einer eiweibarmen Futterration zur Geltung lommen kann, ist die Frage nach dem Einfluß der Amide auf die Milch- und Milchfettbildung verschieden beantwortet worden. Die Verff. haben in den Jahren 1903 und 1904 9 Versuche mit je 3 Ziegen ausgeführt, welche ein Futter erhielten, das bei einem möglichst geringen Eiweißgehalte trotzdem zur Erzielung eines möglichst hohen Milchertrages ausreichte; ein Teil des Eiweißes wurde bei den Versuchen nach dem Stickstoffgehalte und dem Kalorienwerte durch Asparagin ersetzt. Das Grundfutter bestand aus Wiesenheu, Weizenschalen, Leinkuchen, Alcuronat, Kartoffelstärke, Rohrzucker, Kochsalz und Wasser. Aus den Versuchen ergibt sich nach Ausschaltung der individuellen Verschiedenheiten bei den einzelnen Tieren, daß durch den Ersatz von Eiweiß durch Asparagin eine Verminderung der Milchmenge nicht eintritt, daß dagegen der Gehalt der Milch an Trockensubstanz, Fett und Eiweißstoffen abnimmt und daß endlich auch die Zunahme an Lebendgewicht ungünstig beeinflußt wird. Daher kann das Asparagin nicht zu den eigentlichen Nährstoffen gezählt werden, vielmehr sind die Amide nur zur Erzeugung von Wärme, nicht aber von Körpersubstanz befähigt. Andererseits ist aber auch dem Asparagin eine Reizwirkung zuzuschreiben, indem es eine allgemeine Erhöhung des Stoffwechsels hervorzurufen vermag. A. Scholl.

Gustar Fingerling: Untersuchungen über den Einfluß von Reizstoffen auf die Futteraufnahme, Verdaulichkeit und Milchsekretion bei reizlosem und normalem Futter. (Landw. Versuchs-Stat. 1905, 62, 11-180.) - Die Versuche, die an Kaninchen, Ziegen, Hammeln und Milchschafen angestellt wurden, umfassen zwei Reihen. In der ersten Reihe wurde ein reizstoffarmes Futter aus extrahiertem Strohstoff, Troponabfällen, Stärke, Futterkalk und Heuasche für sich und mit Zugaben von Bockshornsamen, Anis, Fenchel, Heu-Destillat und Zucker verabreicht. Hierbei verursachten diese Reizstoffe, abgesehen vom Fenchel, welcher keinen Einfluß ausübte, eine Vermehrung des Futterverzehrs, während ein 